\title{
Contacts for silicon IMPATT and pick-off diodes
}

\author{
N.S. Boltovets, N.M. Goncharuk, V.A. Krivutsa, V.E. Chaika \\ State Scientific \& Research Institute "Orion», 8a Eugene Pottier St., 03057 Kyiv, Ukraine \\ Tel.: (38044)45605 48; E-mail:bms@i.kiev.ua
}

\author{
R.V. Konakova, V.V. Milenin, E.A. Soloviev, M.B. Tagaev, D.I. Voitsikhovskyi \\ Institute of Semiconductor Physics, NAS Ukraine, 45 prospect Nauki, 03028 Kyiv, Ukraine \\ Tel.: (38044) 26561 82; Fax: (38044) 26583 42; E-mail: konakova@eee.semicond.kiev.ua
}

\begin{abstract}
We studied experimentally: (i) the ways to fabricate metal- $n^{+}-\mathrm{Si}$ ohmic contacts with Schottky barriers; (ii) how elemental, structural and phase composition of barrier layers in the contact system, as well as of the barrier layer-semiconductor interface, depend on the formation techniques and conditions; (iii) their evolution at heating and under ${ }^{60} \mathrm{Co} \gamma$-radiation. Some versions of technique to form palladium, titanium, gold barrier layers using thermal and magnetron sputtering, as well as thermionic synthesis, are discussed. We investigated the structure, as well as phase and elemental composition, of both the barrier layers in contact system and barrier layer-semiconductor interface as a function of the formation techniques and conditions, and their evolution under heating and ${ }^{60} \mathrm{Co} \gamma$-radiation. For technological processes of contact system and mesa formation a simulation was performed to determine what contact systems are promising for use in manufacturing technology of silicon IMPATT and pick-off diodes for the millimeter wavelength range. Some conditions have been found that are necessary for production of high-performance contact systems. The heat and radiation tolerance ranges for barrier structures were considered. It was shown that the Si-Ti$\mathrm{TiB}_{2}$-Au contact systems are best suited for production of silicon IMPATT and pick-off diode structures intended for the millimeter wavelength range.
\end{abstract}

Keywords: metal-semiconductor contacts, IMPATT diodes, thermal annealing, simulation

Paper received 27.12.99; revised manuscript received 20.04.00; accepted for publication 06.06.00.

\section{Introduction}

Metal-semiconductor contacts make integral parts of all the semiconductor devices. They may serve as active, as well as passive, device elements [1-3]. In the latter case it is of great importance to reduce the contact effect on the device operating characteristics. For a good ohmic contact resistivity must lie below $10^{-5} \Omega \cdot \mathrm{cm}^{-2}$ [1-3]. Besides, this contact has to block the diffusion of both metal atoms (from the outer part of contact system) to the semiconductor system and semiconductor atoms to the metallization. This can be achieved if there are barrier layers (that remain stable during both the technological cycles when manufacturing diodes and long-term operation) at the metal-semiconductor interface. Such barrier layers (appearing during the technological procedures used for diode manufacturing) are due to high density of surface electron states at the $\mathrm{Si}$ contact surface. The metalsemiconductor barrier increases the contact resistance. However, at high doping level of the layer near the semi- conductor surface, the space charge region (SCR) remains thin enough, so the charge carriers can freely tunnel through it.

Here we present some results of our theoretical and experimental investigations dealing with preparation of radiation- and heat-stable low-resistance metal- $n^{+}-\mathrm{Si}$ ohmic contacts with Schottky barrier (SB) and buffer layers made of metal nitrides, borides and silicides. The contacts studied were intended for silicon IMPATT diodes operating in the millimeter wavelength range and fastoperating (switching time of 2-5 ns) pick-off diodes.

\section{Contact formation technique and methods of investigation}

To form palladium, titanium and gold barrier layers, traditional thermal sputtering was used. The titanium nitride and boride layers were heated with an electron beam using magnetron sputtering in a vacuum (at a residual 


\section{N.S. Boltovets et al.: Contacts for silicon IMPATT and pick-off diodes}

gas pressure of $5 \times 10^{-4} \mathrm{~Pa}$ ) and thermionic synthesis (at a residual gas pressure of $10^{-2} \mathrm{~Pa}$ ) [4-6].

We investigated how elemental, structural and phase composition of both the barrier layers in the contact system and barrier layer-semiconductor interface depend on the formation techniques and conditions; as well as on their evolution at temperature increasing and under ${ }^{60} \mathrm{Co}$ $\gamma$-radiation. To study the barrier contact structure at the initial stages of formation, we used the transmission electronography for thin (10-30 nm) layers and reflection electronography for thick (100-200 nm) layers. (In the latter case the angles of incidence and reflection lied in the 3 to $10^{\circ}$ range.) The structure integrated (over the whole barrier layer thickness) characteristics were studied with $x$-ray diffraction (XRD). The electron and XRD patterns have shown that at rather low $(0.2-0.5 \mathrm{~nm} / \mathrm{s})$ sputtering rates the near-surface barrier layer is an amorphous matrix containing the main phase crystallites (with sizes from 3 to $15 \mathrm{~nm}$ ). When the sputtering rate increases up to $0.6-1.0 \mathrm{~nm} / \mathrm{s}$, then polycrystalline films are formed.

The investigation of the layer morphology after rapid (for $60 \mathrm{~s}$ ) thermal annealing (RTA) in hydrogen atmosphere enabled us to make comparison estimation of the temperature stability for the contact systems studied. RTA simulates, to a first approximation, a pulse overload that might occur in diode structures during their long-term operation.

The boride $\left(\mathrm{TiB}_{2}, \mathrm{ZrB}_{2}\right)$ and nitride ( $\mathrm{TiN}, \mathrm{NbN}$ ) barrier layers on Si remain stable up to temperatures $\leq 600^{\circ} \mathrm{C}$. We investigated and analyzed how the atomic components are distributed over the titanium (niobium) nitridesilicon and titanium (zirconium) boride - silicon structures before and after RTA. It was found that during the nitride (boride) film deposition the corresponding atoms penetrate very deeply into the substrate and interact with Si atoms. As a result, the film-substrate interface moves. The embedded atoms and semiconductor matrix form disordered solid solutions.

RTA at $T=400{ }^{\circ} \mathrm{C}$ is accompanied by structural relaxation in the sputtered contact layer. The transition layer becomes somewhat wider, and its chemical inhomogeneity decreases. The above contact systems are rather tolerant for RTA within the $500-600{ }^{\circ} \mathrm{C}$ temperature range. High-temperature $\left(800-950^{\circ} \mathrm{C}\right) \mathrm{RTA}$ increases the structural-phase nonuniformity of both the sputtered film and contact transition layer. This seems to be related to the occurrence of competing chemical reactions between silicon atoms and metal nitride (boride film components). In this case the silicide and silicate formation is enhanced. This is the physico-chemical reason for high-temperature degradation of contact structures.

Typical concentration depth profiles for the $\mathrm{Au}-\mathrm{TiB}_{\mathrm{x}}-\mathrm{Si}$ contact components taken before and after RTA at $T=400,600$ and $800^{\circ} \mathrm{C}$ and interface morphology after metallization layer removal are shown in Fig. 1 (a-h). One can see that for both initial sample and those annealed at $T=400$ and $600{ }^{\circ} \mathrm{C}$ a correlation exists between the contact layered structure and surface morphology. Topographic nonuniformity of the latter obeys

$S Q O, 3(3), 2000$
Gaussian distribution. RTA at $T=800^{\circ} \mathrm{C}$ substantially disrupts the contact structure. The layers intermix and nonuniform regions appear at the interface. An analysis of topography shows that the metal-silicon interface is a fractal of regularity $D=2.473$ (initial sample) and 2.509 (2.685) after RTA at $T=400(600){ }^{\circ} \mathrm{C}$.

We considered a possibility to use thin (20-50 nm) $\mathrm{Pd}_{2} \mathrm{Si}$ contact layers for fabrication of $\mathrm{Si}-\mathrm{Pd}_{2} \mathrm{Si}-\mathrm{TiN}_{\mathrm{x}}$ $\left(\mathrm{TiB}_{\mathrm{x}}\right)$-Au-type contact systems (here $\mathrm{TiN}_{\mathrm{x}}$ and $\mathrm{TiB}_{\mathrm{x}}$ are buffer layers). Investigation of Pd layers sputtered on the silicon surface has shown that $\mathrm{Pd}$ deposition onto $\mathrm{Si}$ is accompanied with growth of silicide nuclei followed by a silicide layer increase. This process is sustained by diffusion of $\mathrm{Pd}$ and $\mathrm{Si}$ atoms through the growing silicide layer and chemical binding of these atoms. Presence of $\mathrm{SiO}_{2}$ film on the silicon surface does not prevent $\mathrm{Pd}$ atoms to diffuse [7, 8], so it does not affect the silicide phase formation. Further thermal annealing of the contact system at $T=300{ }^{\circ} \mathrm{C}$ for $30 \mathrm{~min}$. and $T=400{ }^{\circ} \mathrm{C}$ for $60 \mathrm{~s}$ in hydrogen atmosphere does not lead to new structural modification of the silicide film. Our physico-chemical investigations have shown that the structure and both phase and elemental composition of the barrier layers studied remained stable at short-term heating up to $600^{\circ} \mathrm{C}$. At temperatures of $800-900{ }^{\circ} \mathrm{C}$ the barrier layer parameters change substantially.

Shown in Fig. 2 are the experimental contact differential resistivity (CDR) vs current density curves taken after RTA at $T=600$ and $800^{\circ} \mathrm{C}$. An analysis of these curves has shown that CDR of high-resistance Si-TiN-Au and Si$\mathrm{TiB}_{2}-\mathrm{Au}$ contacts decreases after RTA at $T=800{ }^{\circ} \mathrm{C}$, while that of low-resistance $\mathrm{Si}-\mathrm{Pd}_{2} \mathrm{Si}$-TiN-Au and $\mathrm{Si}-\mathrm{Ti}$ $\mathrm{TiB}_{2}$-Au contacts grows by 2 to 3 times, RTA at temperatures up to $600{ }^{\circ} \mathrm{C}$ resulted in improvement of characteristics for contacts of both types.

It was found that oxygen impurity could substantially affect the phase transformation character (physico-chemical characteristics of barrier layers, contact system modifications during high-temperature processing [10-12]). However, this fact does not prevent to use such barrier layers in the microwave diode contact systems with mesas.

Our investigations of combining the technology of contact system manufacturing with technological cycle of diode mesa production have shown that minimum etch undercutting of the $\mathrm{Si}-\mathrm{Pd}_{2} \mathrm{Si}$-TiN-Au contact system occurs when a silicon mesa (diameter of $30-50 \mu \mathrm{m}$, height of $10-15 \mu \mathrm{m}$ ) is formed. One can avoid such an effect when mesas of bigger (over $60 \mathrm{~mm}$ ) diameter are formed using the $\mathrm{Si}-\mathrm{Pd}_{2} \mathrm{Si}-\mathrm{Ti}-\mathrm{TiB}_{2}-\mathrm{Mo}$-Au contact system.

Exposition to ${ }^{60} \mathrm{Co} \gamma$-radiation (dose range from $10^{3}$ to $10^{5} \mathrm{~Gy}$ ) did not change CDR of the $n^{+}-\mathrm{Si}-\mathrm{Pd}_{2} \mathrm{Si}-\mathrm{TiN}-$ Au systems. It reduced the statistical spread of the diode structure parameters. This is related to the radiation-induced defect gettering in contacts [9].

\section{Model for contacts}

We have performed computer simulation of the charge carrier transport in metal- $n^{+}-\mathrm{Si}$ contacts with a rather 
N.S. Boltovets et al.: Contacts for silicon IMPATT and pick-off diodes

a)
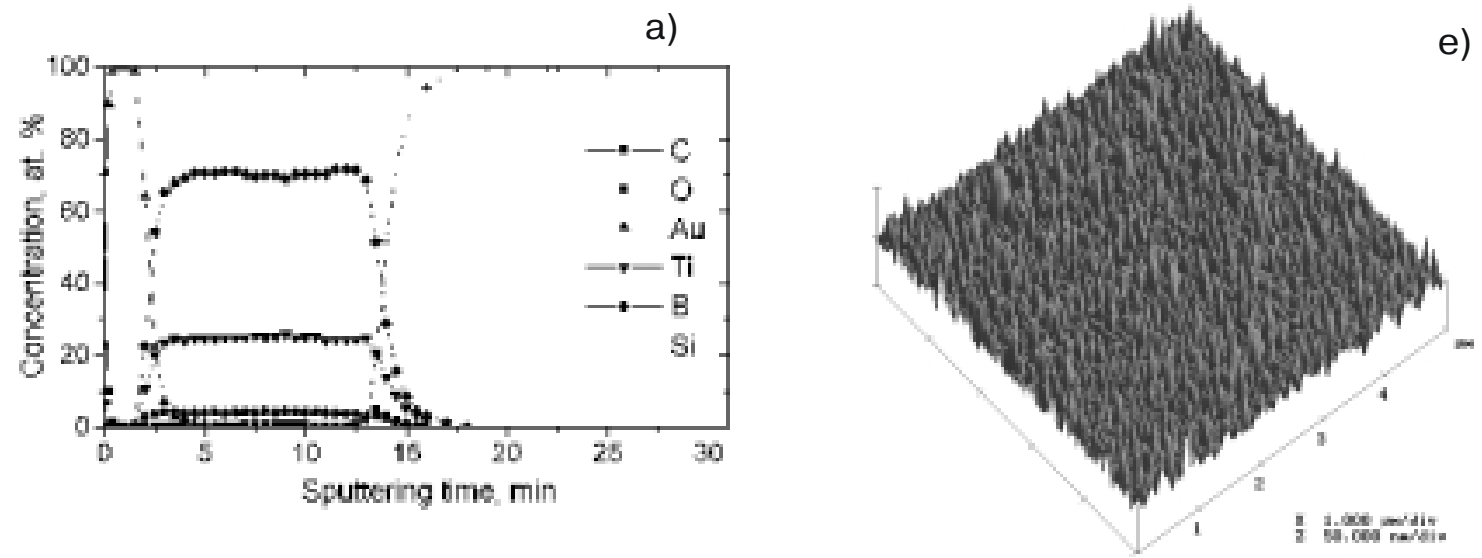

b)

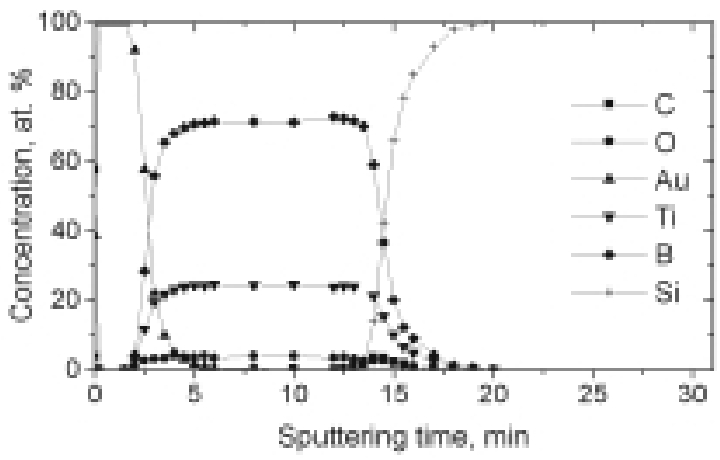

g)

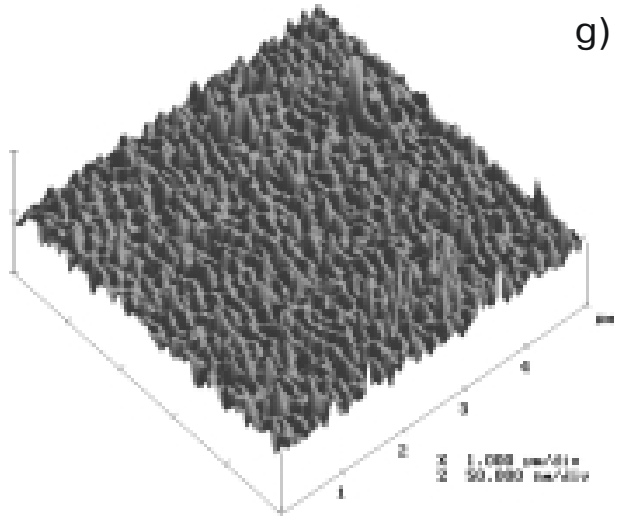

c)

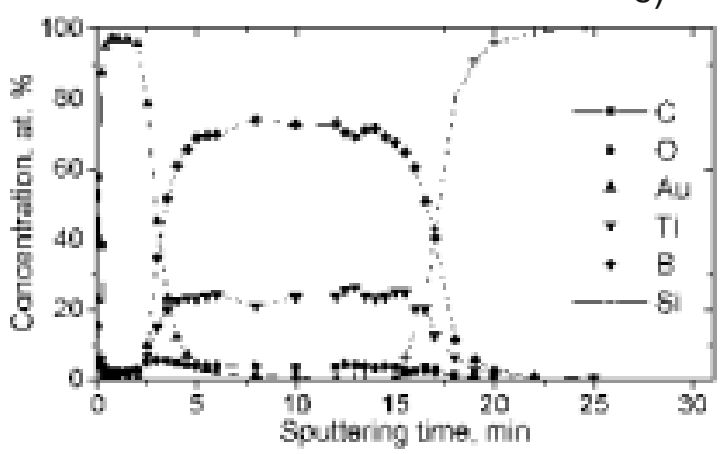

f)

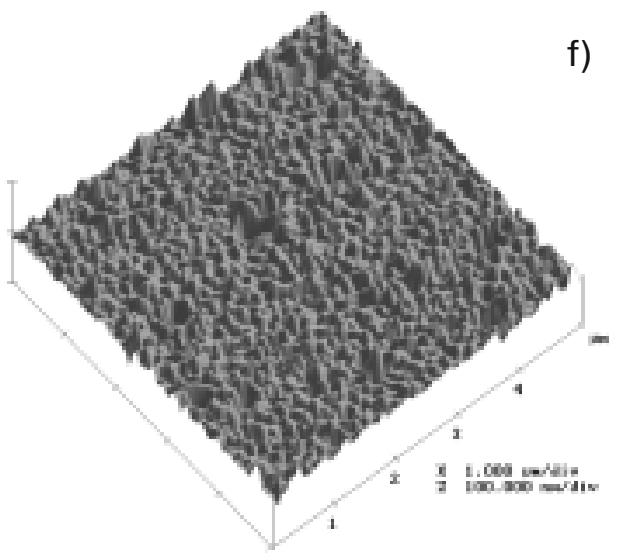

d)
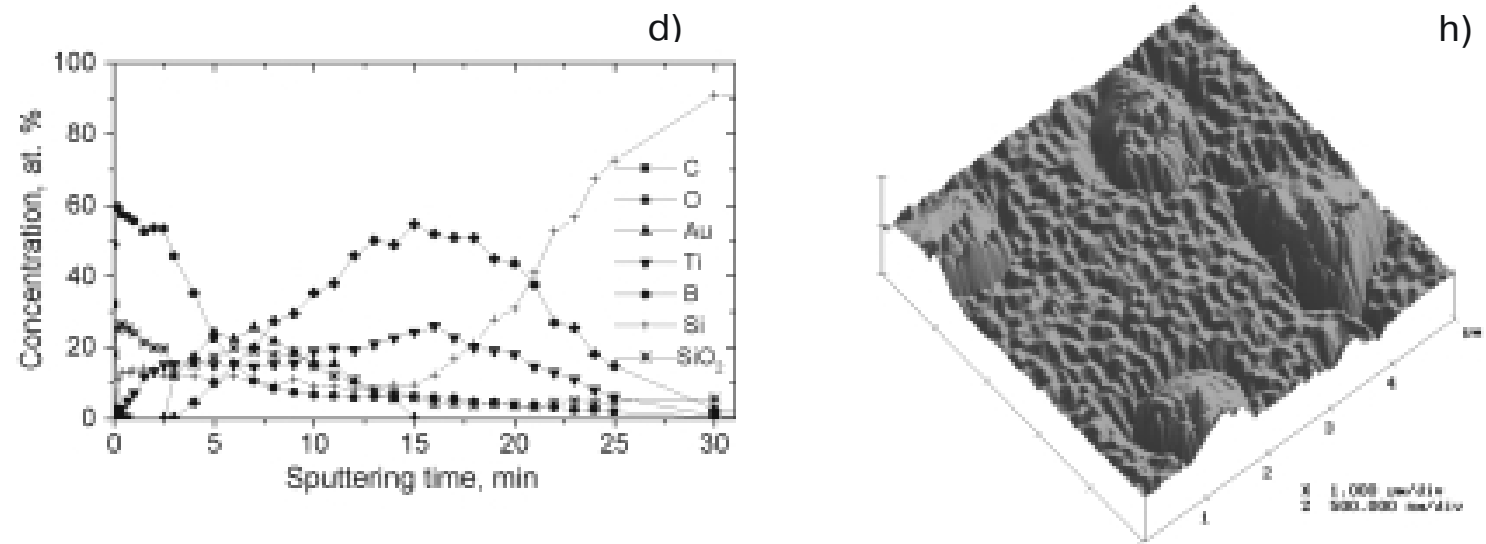

Fig. 1. Concentration depth profiles for $\mathrm{Au}-\mathrm{TiB}_{2}-\mathrm{Si}$ contacts before (a) and after annealing at $T=400$ (b), 600 (c) and $800{ }^{\circ} \mathrm{C}$ (d) in hydrogen atmosphere for $60 \mathrm{~s}$; metal-Si interface morphology of the corresponding structure after removal of metallization layers (e-h). 


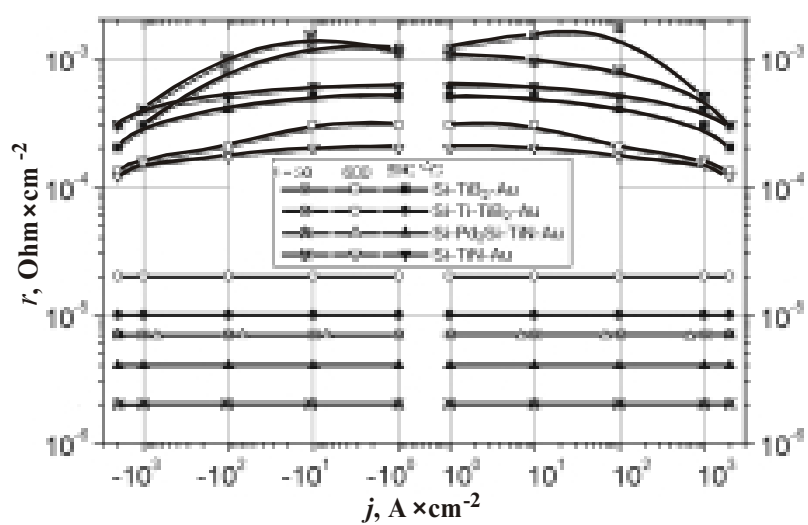

Fig. 2. Experimental contact differential resistivity vs current density curves taken for initial and annealed at $T=600$ and $800{ }^{\circ} \mathrm{C}$ contacts. (The donor concentration $N_{d}$ in the starting wafers was $2 \times 10^{19} \mathrm{~cm}^{-3}$.)

high (donor concentration $N_{d} \geq 2 \times 10^{19} \mathrm{~cm}^{-3}$ ) doping level of the semiconductor near-surface region. The total current through the contact was calculated as a sum of two components: thermionic

$J_{t e}=\frac{A^{*} T^{2}}{k} \int_{0}^{\infty} D_{d}(\xi) e^{-\frac{\xi}{k T}}\left[e^{\frac{-\left(V_{B}-\mu\right)}{k T}}-e^{\frac{-q \varphi_{B}}{k T}}\right] d \xi$

and tunnel

$J_{t}=\frac{A^{*} T}{k} \int_{0}^{V_{B}-q U} D_{d}(\varepsilon) D_{b}(\varepsilon)\left(f_{s}-f_{m}\right) d \varepsilon$

[1]. Here $\varphi_{B}$ is the SB height with account for its decrease due to image force of an adjacent (metal or insulator) region; $A^{*}$ is the effective Richardson constant for electrons leaving silicon in the [111] direction; $\varepsilon$ is the tunneling electron energy; $\xi$ is the excess of the emitted electron energy over the potential barrier in semiconductor SCR; $V_{B}$ is band bending in the near-contact SCR; $f_{s}$ $\left(f_{m}\right)$ is the electron distribution function in the silicon (metal) conduction band; $k$ is Boltzmann constant; $D_{d}$ and $D_{b}$ are the quantum penetration probabilities for the potential barriers of an insulating layer (IL) and SB, respectively. They were calculated numerically using the following expression:

$D_{d, b}=\exp \left\{-\left[\frac{\left(8 m^{*}\right)^{\frac{1}{2}}}{\hbar}\right] \int_{A}^{B}[q \varphi(x)-\varepsilon]^{\frac{1}{2}} d x\right\}$

In (3) we took into account changes in the height and form of both (Schottky and IL) barriers due to the image force of the adjacent regions [3]. $A$ and $B$ are the tunneling electron turning points for every value of its energy $\varepsilon$; $q\left(m^{*}\right)$ is the electron charge (effective mass) for the [111] direction in silicon (the electron mass in IL was taken equal to $\left.m^{*}\right) ; h$ is reduced Planck's constant; $q \varphi(x)$ is the potential barrier height at point $x$.

The SB and electric field in SCR were calculated by solving numerically a set of two differential equations. The first of them was derived analytically after single integration of the one-dimensional Poisson's equation for Fermi distribution of electrons in the heavily doped semiconductor substrate:

$$
\begin{aligned}
& \frac{\partial \varphi}{\partial x}=\left\{\left(\frac{n_{i}}{n_{0}}-\frac{n_{0}}{n_{i}}\right) \beta \varphi-\right. \\
& -\left[N_{v} \int_{0}^{\infty} \sqrt{\varepsilon} \ln \left|\frac{1+\exp \left[-\left(\varepsilon+\varepsilon_{g}+\mu-q \varphi\right) / k T\right]}{1+\exp \left[-\left(\varepsilon+\varepsilon_{g}+\mu\right) / k T\right]}\right| d \varepsilon+\right. \\
& \left.+N_{c} \int_{0}^{\infty} \sqrt{\varepsilon} \ln \left|\frac{1+\exp [-(\varepsilon-\mu+q \varphi) / k T]}{1+\exp [-(\varepsilon-\mu) / k T]}\right| d \varepsilon\right] \times \\
& \left.\times \frac{2}{n_{i} \sqrt{\pi}}\right\}^{1 / 2}\left(\beta L_{d}\right)^{-1}
\end{aligned}
$$

The second one is the equation for the electric field $F$ in SCR:

$F=-\frac{d \varphi}{d x}$.

Here $\mathrm{j}$ is the electrostatic potential; $n_{i}$ and $n_{0}$ are the intrinsic charge carrier concentration and conduction electron concentration in silicon outside SCR; $\mu$ is Fermi energy for conduction electrons in silicon (it was found by numerical calculation of Fermi integral for every value of doping level in the semiconductor near-surface region; $\varepsilon_{g}$ is the silicon band gap value; $\beta=q / k T ; N_{c}\left(N_{v}\right)$ is the effective density of states in the conduction (valence) band; $L_{d}$ is Debye length for intrinsic charge carriers in silicon.

At calculations of the electrostatic potential and electric field in SCR of SB as a function of coordinate $x$, Boltzmann distribution is usually used for free charge carriers even in the case of a heavily doped semiconductor. This may be done because the electron energy at a point $x$ of SCR increases in the electric field of SB by a rather big amount $q \varphi(x)$, and so

$(\varepsilon+q \varphi-\mu)>>k T$,

i.e., the Boltzmann approximation for the free electron distribution function $f_{s}(\varepsilon)$ in the semiconductor SCR is valid. Indeed, our calculations of the CDR for tight contacts on a heavily doped substrate have shown that its values obtained using the Fermi and Boltzmann approximations differ by less than $10 \%$. However, presence of an intermediate IL in actual contacts leads to SB drop 


\section{N.S. Boltovets et al.: Contacts for silicon IMPATT and pick-off diodes}

(that is bigger for thicker insulating layers). It also increases a contribution to the total current from electron emission over SB with further electron tunneling through the IL barrier. For actual contacts such a contribution results in much greater (as compared to the case of tight contacts) drop of CDR values calculated using Fermi approximation for $f_{s}(\varepsilon)$ than that calculated using Boltzmann approximation for $f_{s}(\varepsilon)$. An account of the fact that the free charge carrier distribution in SCR is of Fermi-type becomes even more important when temperature and SB height go down and the doping level of nearcontact SCR increases.

The set of equations (1) and (2) was solved at the following boundary conditions: both the electric field and potential at the boundary between SCR and semiconductor neutral region are zero; the electrostatic potential at the boundary between semiconductor and metal (or insulator) is the applied voltage drop on semiconductor SCR plus SB height at zero voltage. The electric field in IL was determined from the condition of the electric displacement continuity at the Il-semiconductor interface.

\section{Results of calculations}

For contacts with $N_{d}=2 \times 10^{19}, 5 \times 10^{19}, 10^{20}$ and $5 \times 10^{20} \mathrm{~cm}^{-3}$ we have calculated $I-V$ curves and CDR vs current density, $\rho(j)$, curves at temperatures $T=213,300$ and $500 \mathrm{~K}$. The SB height values were those characteristic of Ti-Si, $\mathrm{Pd}-\mathrm{Si}, \mathrm{Au}-\mathrm{Si}$ and Pt-Si contacts that are used in manufacturing technology of microwave semiconductor devices.

Shown in Fig. 3 are theoretical CDR vs current density curves for tight Ti-Si contacts. The doping levels of the semiconductor near-contact SCR and temperatures are $2 \times 10^{19}$ (curves $1-3$ ), $10^{20}$ (curves $1 \mathrm{a}-3 \mathrm{a}$ ) and $213 \mathrm{~K}$ (curves 1, 1a), $300 \mathrm{~K}$ (curves 2-2c), $500 \mathrm{~K}$ (curves 3-3b), respectively. One can see that CDR is constant at forward and reverse currents from a rather large range near zero current. At increase of temperature and doping level of semiconductor SCR this range expands, while CDR goes down. Beyond the above range $C D R$ decreases with both forward and reverse currents. For reverse currents there is a small peak at the boundary of this range. This peak becomes less high and shifts to higher current densities with the doping level; it shifts to lower current densities with temperature. This, along with the fact that voltage close to $\mu / q$ corresponds to the limiting forward current value, evidences that the above peak is related to the mean conduction electron energy in the semiconductor. At extremely high $\left(N_{d} \geq 10^{20} \mathrm{~cm}^{-3}\right)$ doping level of SCR the contacts are ohmic, since CDR remains constant and rather low $\left(\leq 5 \times 10^{-6} \mathrm{~W} \cdot \mathrm{cm}^{2}\right)$ over the temperature range studied and wide current density range (for $N_{d} \geq 10^{20} \mathrm{~cm}^{-3}$ the current density is higher than $\left.10^{5} \mathrm{~A} / \mathrm{cm}^{2}\right)$.

Shown in Fig. 4 are contact energy diagrams for several applied voltages. They serve to explain our interpretation of the nature of linear portion of $I-V$ curves corresponding to low values of forward and reverse currents. The diagrams a-e correspond to zero applied voltage, low reverse voltage and forward voltages below, equal to

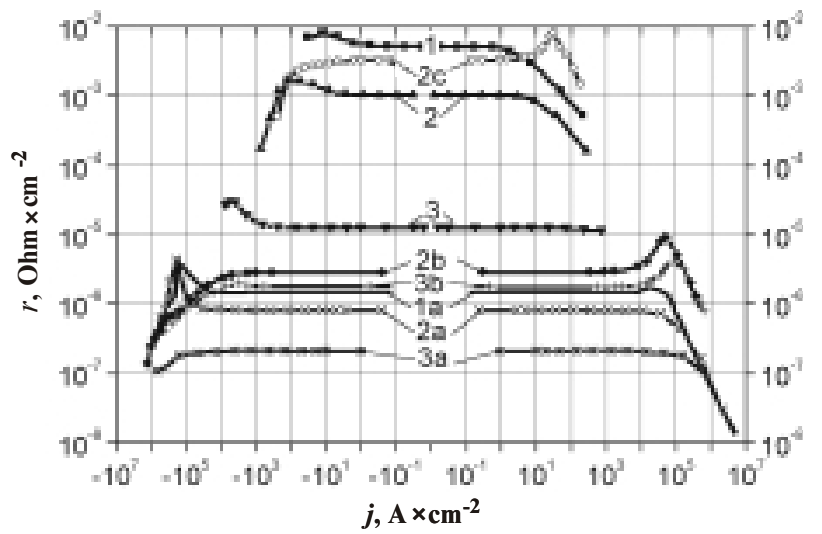

Fig. 3. Calculated differential resistivity vs current density curves for tight contacts (1-3, 1a-3a) and actual Si-Ti-Au contacts with $N_{d}=2 \times 10^{19}(1,2,2 \mathrm{c}, 3)$ and $10^{20} \mathrm{~cm}^{-3}(1 \mathrm{a}, 2 \mathrm{a}, 2 \mathrm{~b}, 3 \mathrm{a}, 3 \mathrm{~b})$ at $T=213$ $(1,1 \mathrm{a}), 300(2,2 \mathrm{a}, 2 \mathrm{~b}, 2 \mathrm{c})$ and $500 \mathrm{~K}(3,3 \mathrm{a}, 3 \mathrm{~b})$; the insulating layer thickness $H=0.5 \mathrm{~nm}(2 \mathrm{~b}, 2 \mathrm{c}, 3 \mathrm{~b})$.

and over Fermi potential $V_{F}$ of conduction electrons in Si. We performed an analysis of these diagrams and the expression for tunnel component of the current through a contact (this component determines the total current value at rather high doping levels of SCR that were considered) and made the following conclusions. Over a narrow range (near zero) of forward and reverse voltages the SB penetration probability depends only slightly on voltage applied. The current growth is predominantly due to range expansion for the conduction electrons (that tunnel to the adjacent region) of semiconductor (at forward voltage) or metal (at reverse voltage). The width of this range depends linearly on the voltage applied (for forward voltages $U \leq V_{F}$ and any reverse voltage). These factors result in the $I-V$ curve linearity over the above voltage range and CDR independence from current density over the corre-

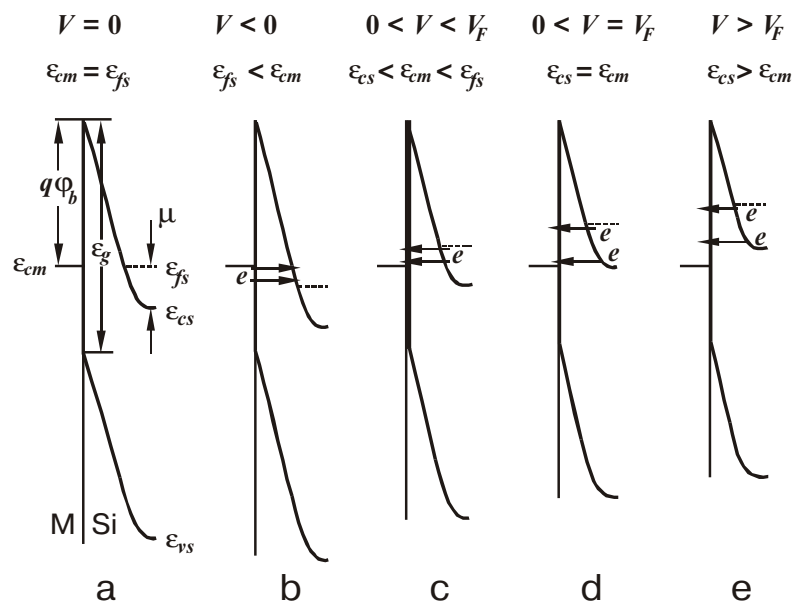

Fig. 4. Energy diagrams for a tight contact at different voltages applied: zero (a); low reverse (b); forward below (c), equal to (d) and over (e) Fermi potential $V_{F}$ in $\mathrm{Si}$. 


\section{N.S. Boltovets et al.: Contacts for silicon IMPATT and pick-off diodes}

sponding current range. The widths of these ranges increase when $N_{d}$ grows or $\varphi_{B}$ decreases, and (to a lesser extent) when temperature grows.

Shown in Fig. 5 are the calculated $I-V$ curves for Ti-Si contacts with $N_{d}=2 \times 10^{19}, 5 \times 10^{19}, 10^{20}$ and $5 \times 10^{20} \mathrm{~cm}^{-3}$ (curves 1-1c) and Pd-Si, Au-Si, Pt-Si contacts with $N_{d}=2 \times 10^{19} \mathrm{~cm}^{-3}$ (curves $2-4$ ) at $T=300 \mathrm{~K}$. One can see that when $\varphi_{B}$ grows, the $I-V$ curves go down over all the voltage range studied except rather high reverse voltages. At its threshold value, $V_{t}=\varepsilon_{g}-\varphi_{\mathrm{B}}$ (the bigger $\varphi_{\mathrm{B}}$, the higher $V_{t}$ ), the current abruptly increases with the reverse voltage. The range of voltages $U>V_{t}$ corresponds to a strong inversion of the space charge in the semiconductor near-surface layer adjacent to metal. An abrupt increase of the free holes charge (and, accordingly, the SCR electric field) with reverse voltage under this conditions leads to a considerable SB narrowing for electrons that tunnel from metal to semiconductor. This results in an abrupt change of contact resistance and $I$ - $V$ curve slope. Along with this tunneling mechanism (that is predominant at $U<V_{t}$ ), two more mechanisms of current flow through contact appear and increase their roles. The first of them is electron band-to-band tunneling (from the valence band to the conduction band of the semiconductor). The second mechanism is the hole injection from the semiconductor valence band to metal. However, these two mechanisms are of essence at a rather high reverse voltage (the lower SB, the higher this reverse voltage value). So we do not consider them in our theoretical model for contacts.

An analysis of the $\rho(j)$ curves for actual contacts with IL $0.5 \mathrm{~nm}$ thick obtained at $N_{d}=2 \times 10^{19} \mathrm{~cm}^{-3}, T=300 \mathrm{~K}$ (Fig. 3, curve 2c) and $N_{d}=10^{20} \mathrm{~cm}^{-3}, T=300,500 \mathrm{~K}$ (Fig. 3, curves 2b, 3b) shows that presence of IL leads to a CDR increase. The higher are temperature and semiconductor doping level, the greater is this increase. When IL becomes thicker, then the CDR dependence on temperature and SCR doping level becomes weaker. The CDR dependence on current density has a small peak at a forward (contrary to the case of tight contacts) current.

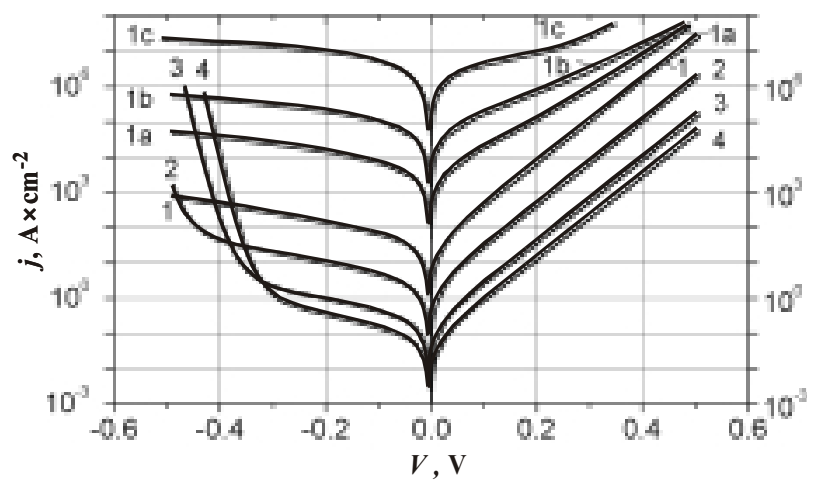

Fig. 5. Calculated $I-V$ curves for tight Ti-Si contacts with $N_{d}=$ $2 \times 10^{19}(1), 5 \times 10^{19}(1 \mathrm{a}), 10^{20}(1 \mathrm{~b})$ and $5 \times 10^{20} \mathrm{~cm}^{-3}$ (1c) and Pd-Si (2), Au-Si (3), Pt-Si (4) contacts with $N_{d}=2 \times 10^{19} \mathrm{~cm}^{-3}$ at $T=300 \mathrm{~K}$.

$S Q O, 3(3), 2000$
This peak lies at the boundary between the two portions of the $\rho(j)$ curve (namely, those dependent on current and independent from it). The peak height grows at temperature decrease and increase of the semiconductor SCR doping level. One may assume that this peak corresponds to changing the current flow mechanism: predominant tunneling through both (IL and Schottky) barriers changes for a mixed mechanism that involves, along with the above, also over-the- SB emission followed by tunneling through IL.

\section{Experimental studies of the contact electrophysical parameters}

To study experimentally the contact electrophysical parameters, we used standard silicon wafers with resistivity of $0.003 \Omega \cdot \mathrm{cm}$. They served as substrates to epitaxially grow active areas of microwave diode structures, as well as silicon test diode structures with thin layers of borides or nitrides (serving as barrier materials). At low frequencies the measurements were performed on direct mesas, while at high frequencies they were performed on the diode chips built in high-frequency packages. We studied experimentally $I$ - $V$ curves and CDR vs current (or voltage) curves at current densities from 1 to $10^{4} \mathrm{~A} / \mathrm{cm}^{2}$. The frequency range was $1 \mathrm{MHz}-12 \mathrm{GHz}$, and temperature range of contact system functioning was from 213 to $500 \mathrm{~K}$.

The experimental CDR vs current density curves taken at $T=300 \mathrm{~K}$ for Si-TiN-Au, Si- TiB $2-\mathrm{Au}, \mathrm{Si}-\mathrm{Ti}-\mathrm{TiB}_{2}-\mathrm{Au}$ contact systems (curves $1,2,4$ ) and at $T=300,213$, $500 \mathrm{~K}$ for Si-Ti-Au contact system are shown in Fig. 6. The donor concentration in the initial wafers was $N_{d}=$ $2 \times 10^{19} \mathrm{~cm}^{-3}$. At $T=300 \mathrm{~K}$ and current density below $10^{4} \mathrm{~A} / \mathrm{cm}^{2} \mathrm{CDR}$ of contacts with $\mathrm{TiB}_{2}$ and $\mathrm{TiN}$ barrier layers depends on current and lies from $10^{-3}$ to $10^{-4} \Omega \cdot \mathrm{cm}^{2}$. That for $\mathrm{Si}-\mathrm{Ti}-\mathrm{Au}$ contacts lies within narrower range (from $2 \times 10^{-5}$ to $6 \times 10^{-5} \Omega \cdot \mathrm{cm}^{2}$ ) and does not depend on current. For $\mathrm{Si}-\mathrm{Ti}-\mathrm{TiB}_{2}-\mathrm{Au}$ system $\mathrm{CDR}$ is about $7 \times 10^{-6} \Omega \cdot \mathrm{cm}^{2}$. For the contacts with $\mathrm{TiB}_{2}$ and TiN bar-

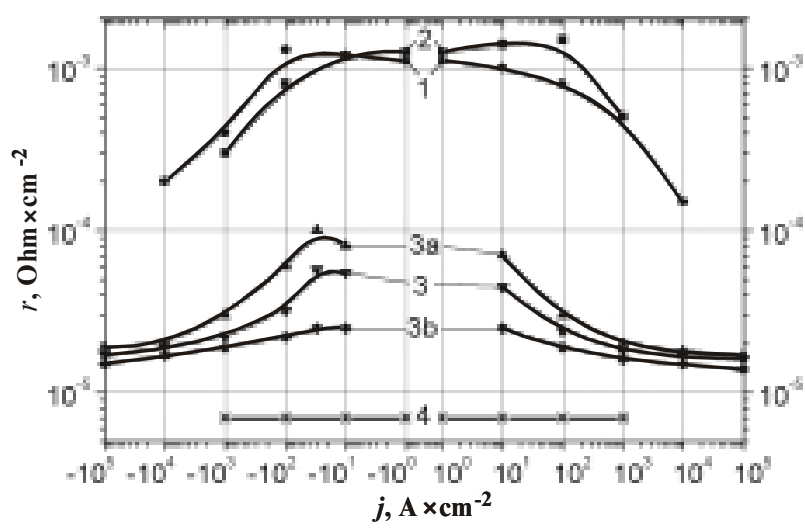

Fig. 6. Experimental differential resistivity vs current density curves taken for Si-TiN-Au (1), Si-TiB $2-\mathrm{Au}$ (2), Si-Ti-TiB $2-\mathrm{Au}$ (4) contact systems with $N_{d}=2 \times 10^{19} \mathrm{~cm}^{-3}$ at $T=300 \mathrm{~K}$ and Si-TiAu contact system at $T=300$ (3), 213 (3a), $500 \mathrm{~K}(3 \mathrm{~b})$. 


\section{N.S. Boltovets et al.: Contacts for silicon IMPATT and pick-off diodes}

rier layers the experimental $\rho(j)$ curves are close to the theoretical ones, while for $\mathrm{Si}-\mathrm{Ti}$-Au contacts they satisfactorily agree with the theoretical curves at $N_{d}=10^{20} \mathrm{~cm}^{-3}$. The last fact may be explained by the appearance at the silicon surface of a thin layer of recrystallized silicon with free charge carrier concentration over the doping level of the initial Si wafer [8]. This is evidence that a possibility exists of further CDR reduction in contact systems using the appropriate manufacturing technology.

The effect of temperature on both the experimental CDR and its current dependence is less pronounced for contacts with lower resistivity. This agrees with the theoretically obtained result that the temperature effect decays when the doping level in the semiconductor nearsurface region is increased. In this case CDR decreases slightly and in the same degree for all the values of forward and reverse currents (remaining independent from current). For Si-Ti-Au contact system, when temperature is increased from 213 up to $500 \mathrm{~K}$, the CDR drop is maximum (by a factor of four) near the peak of the $\rho(j)$ curve and decreases when moving from the peak (see Fig. 6, curves $3,3 \mathrm{a}, 3 \mathrm{~b})$. Therefore the peak of the $\rho(j)$ curve is smoothing out when temperature grows; at $T=500 \mathrm{~K}$ it is pronounced but slightly. The CDR temperature dependence is in quantitative agreement with that predicted theoretically. However, the extent of experimental CDR changing with temperature is less than that calculated theoretically.

For Si-Ti-Au contacts we studied experimentally the CDR dependence on the alternating current frequency. From an analysis of $\rho(j)$ curves taken at different frequencies it follows that the extent of CDR frequency dependence increases when current goes down. So the only peak of the $\rho(j)$ curve existing at frequencies $f \leq 1.5 \mathrm{MHz}$ transforms to two peaks when frequency is increased: a peak for forward current and another one for reverse current (a minimum lies at a current close to zero). At frequencies $f \geq 10 \mathrm{GHz}$ both peaks disappear and contacts are ohmic: CDR is below $10^{-5} \Omega \cdot \mathrm{cm}^{2}$ over all the ranges of forward and reverse currents. The experimentally obtained regularities in CDR frequency dependence can be explained within the contact model developed in [2]. This model accounted for both an insulating gap and surface states. The effect of the latter is the greatest (i.e., $d \rho / d f$ is maximum) for frequencies $f \geq 1.5 \mathrm{GHz}$ at both current and voltage close to zero; this effect becomes less pronounced when current (forward or reverse).

\section{Conclusions}

The performed computer simulation of charge transport in metal-semiconductor contacts with, as well as without, an insulating layer, experimental modeling of technological procedures used to form contact systems and investigation of their electrophysical parameters enable us to draw the following conclusions:

1. To prepare ohmic contact systems to heavily doped regions of a semiconductor, one has to form (at metalsemiconductor interface) a transition recrystallized or overdoped $\left(\geq 10^{20} \mathrm{~cm}^{-3}\right)$ layer without insulating interlayer (or with it, but its thickness must be less than $0.5 \mathrm{~nm}$ ). In this case contact differential resistivity is $\leq 5 \times 10^{-6} \Omega \cdot \mathrm{cm}^{2}$ over the $213-500 \mathrm{~K}$ temperature range and does not depend on current density $j$ up to $j \approx 10^{5} \mathrm{~A} / \mathrm{cm}^{2}$.

2 . Barrier layers of the contact systems studied retain stable structural and phase characteristics after shortterm (60 s) thermal annealing at temperatures below $600{ }^{\circ} \mathrm{C}$ and ${ }^{60} \mathrm{Co} \gamma$-irradiation (doses up to $10^{5} \mathrm{~Gy}$ ).

The $\mathrm{Si}-\mathrm{Ti}-\mathrm{TiB}_{2}-\mathrm{Au}$ contact systems seem the most promising for silicon IMPATT and pick-off diode structures operating in the millimeter wavelength range.

\section{References}

1. S.M. Sze, Physics of Semiconductor Devices, WileyInterscience Publ. John Wiley and Sons, New York-Chichester- Brisbane-Toronto-Singapore (1981).

2. V.I. Strikha, Theoretical Foundations of the Metal-Semiconductor Contact Operation (in Russian), Naukova Dumka, Kiev (1974).

3. E.H. Rhoderick, Metal-Semiconductor Contacts, Clarendon Press, Oxford (1978)

4. N.M. Goncharuk, V.E. Chaika, N.S. Boltovets, V.A. Krivutsa, Ohmic contacts for microwave semiconductor devices (in Russian), in Abstracts th $^{\text {th }}$ Intern. Crimean Conf. "Microwave Engineering and Telecommunication Technologies», Crimea, Ukraine, pp. 151-152 (1998).

5. D.I. Voitsikhovskyi, R.V. Konakova, V.V. Milenin, E.A. Soloviev, M.B. Tagaev, O.D. Smijan, N.S. Boltovets, N.M. Goncharuk, V.A. Krivutsa, V.E. Chaika, Effect of short-term thermal annealing on the characteristics of ohmic contacts to silicon microwave diodes (in Russian) // Fiz. Khim. Obrab. Mater. № 5, pp. 80-84 (1999).

6. N.S. Boltovets, G.M. Verimeichenko, V.N. Ivanov, V.A. Krivutsa, G.V. Beketov, D.I. Voitsikhovskyi, R.V. Konakova, V.V. Milenin, E.A. Soloviev, G.N. Kashin, O.D. Smijan, A.I. Senkevich, Fabrication techniques and properties of $\mathrm{TiN}_{\mathrm{x}}$, $\mathrm{TiB}_{\mathrm{x}}-\mathrm{GaAs}$ and $\mathrm{TiN}_{\mathrm{x}}, \mathrm{ZrB}_{\mathrm{x}}-\mathrm{Si}$ contacts (in Russian), in Proc. $3^{\text {rd }}$ Intern. Sympos. "Vacuum Technologies and Equipment», Kharkov, Ukraine, August 22-24, 1999, 1, pp. 39-48.

7. Thin Films: Interdiffusion and Reactions, Eds. J.M. Poate, K.N. Tu, J.W. Mayer, Wiley-Interscience Publ. John Wiley and Sons, New York-Chichester-Brisbane-Toronto-Singapore (1978).

8. K. Gershinskii, A.V. Rzhanov, E.I. Cherepov, Thin-film silicides in electronics (in Russian) // Mikroelektronika 11 (2), pp. 83-94 (1982).

9. Yu.S. Kleinfeld, R.V. Konakova, V.F. Sinkevich, A.A. Pavlenko, The influence of radiation-stimulated gettering on the reliability of silicon device structures // J. Electrical Eng. 44 (6), pp.177-178 (1993).

10. O.B. Yatsenko, L.Ya. Tverdokhlebova, E.A. Sad'egov, Some structural features of the $\mathrm{TiSi}_{2}$ films obtained using annealing in vacuum (in Russian) // Neorgan. Mater. 28 (3), pp. 536540 (1992).

11. A.M. Chaplanov, A.N. Shibko, Changes in composition factor and electrical properties of Ti films on Si at thermal annealing and laser action (in Russian) // Neorgan. Mater. 31 (9), pp. 1211-1215 (1995).

12. I.S. Nuprienok, A.N. Shibko, Titanium-silicon phase transformations at a combined treatment (in Russian) // Poverkhnost № 2, pp. 45-49 (1997).

13. E.P. Domashevskaya, Yu.A. Yurakov, N.A. Rumyantseva, L.Ya. Tverdokhlebova, E.A. Turenko, S.N. Sklyarov, Investigations of physico-chemical and electro-radiation properties of $\mathrm{Ni}(\mathrm{Ti}) \mathrm{Si}$ and $\mathrm{Ni}(\mathrm{Ti}) \mathrm{Ti} / \mathrm{Si}$ thin-film structures (in Russian) // Mater. Elektron. Tekhn. № 1, pp. $45-49$ (1998). 